\title{
SOME PLASTIC OPERATIONS ABOUT THE LIDS AND SOCKET*
}

\author{
BY
}

Percival J. Haty,

OPHTHALMIC SURGEON, ROYAL HOSPITAL, SHEFFIELD.

\section{I.-Apostasis and Ectropium of the Lower Lid}

We sometimes meet with cases where the lower lid stands away from the globe, with or without slight ectropium of the margin of the lid? They are seen after recent burns. and wounds, and are accompanied by constant lacrimation, owing to exposure of the conjunctiva and lack of apposition of the punctum lacrimale to the globe. Similar conditions are also found among elderly people in whom the tissues are wanting in tone.

The procedures usually adopted to try to bring about contact of the lid and globe are either to excise a triangular portion of skin at the inner canthus, or to shorten the lid by removing a triangular

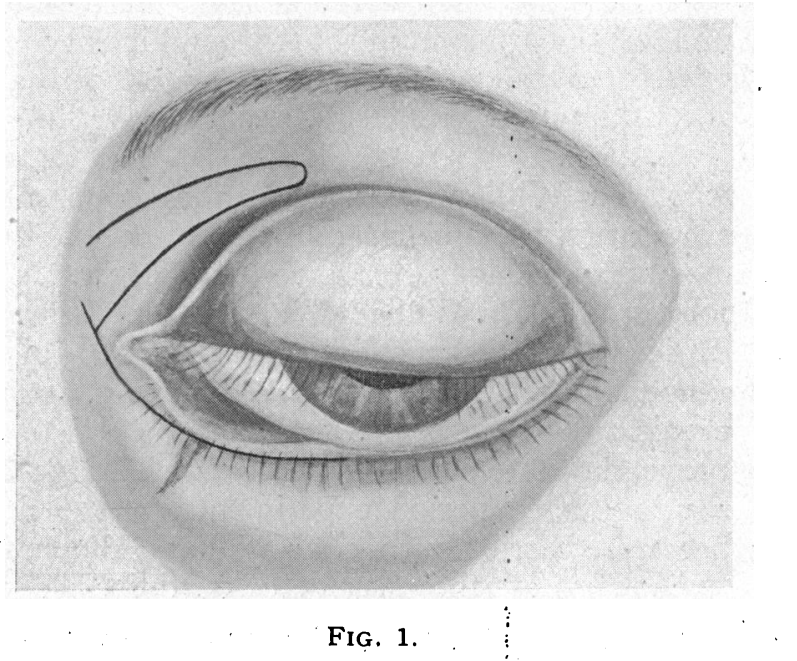

portion of the "cartilage." The former generally fails to achieve the end, and the latter is a delicate and rather tedious operation. I prefer to work as follows :

My aim is to provide a sling with the aid of a pedicle flap taken from the loose tissue between the upper lid and the eyebrow (see Figs. 1 and 2). The arrangement not only tends to improve the position of the lid as a whole, but it also helps to invert the punctum.

* Read before the Midland Ophthalmological Society, March 30, 1920. 
The base of the flap is placed above a line drawn inwards from the internal canthus (or outwards from the external canthus). The height above this level depends upon the extent to which the

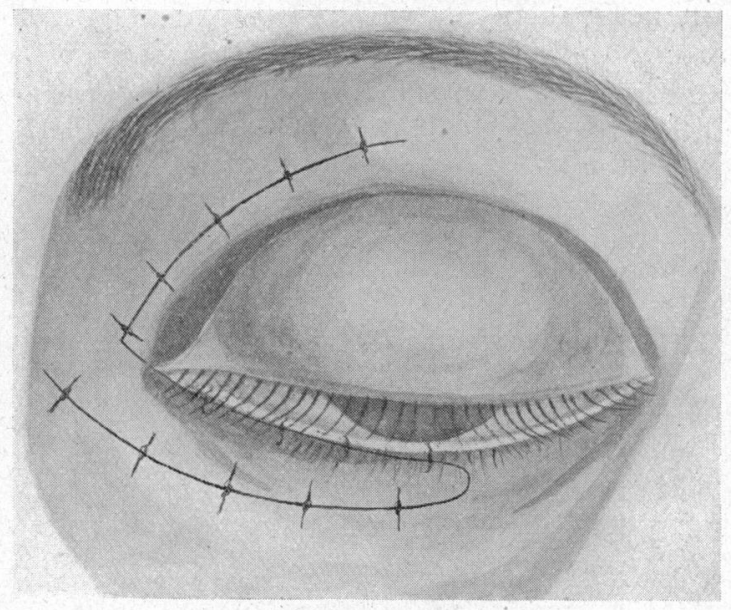

FIG. 2.

lid has to be lifted. It is better to put it too high than too low. $\frac{3}{4}$-inch will meet an average case. From here the flap extends upwards, above and parallel to the principal fold of the upper lid,

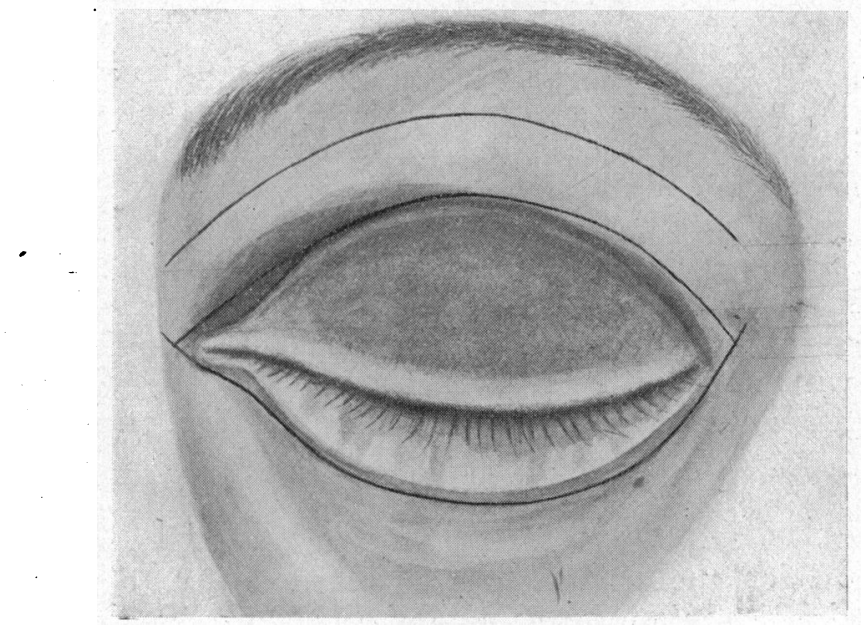

Fig. 3.

length and width of course corresponding to the size and character of the defect to be remedied. 
From the base of the flap an incision is carried downwards along the margin of the lower lid. The skin is freely undermined, care being taken to keep as near the surface as possible, and any cicatricial tissue is divided sufficiently to allow the lid to lie easily against the globe of the eye.

Before turning the flap down into position it is well to incise it slightly on the lower side at the base, and to undermine the latter a little to facilitate turning. The flap should be kept thin to obviate

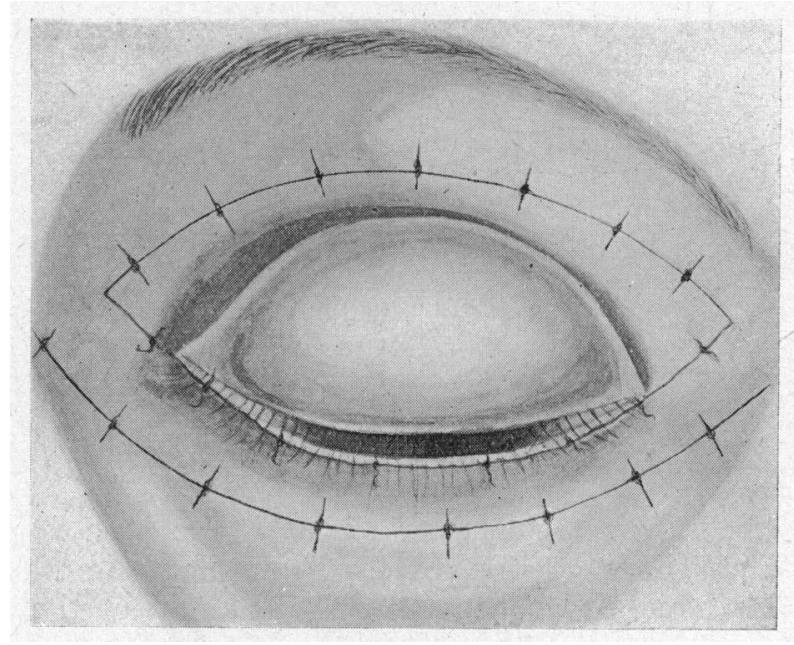

FIG. 4.

an unsightly bulge when is is placed in its new position. Finally, the lid is hitched up, the flap put moderately on the stretch and sutured, silk being used near the lid margin, and horse hair or gossamer silkworm gut elsewhere.

The operation does not interfere in the least with the free movement of the upper lid, and the scars are quite invisible in a few weeks. It can be applied to both ends simultaneously if desired, and if necessary a continuous strip can be taken from the position indicated, with a base at each end, and brought down to form a complete new covering for the lower lid (Figs. 3 and 4).

\section{II.-Contracted Socket}

\section{(a) Cicatricial Bands}

Cases in which the artificial eye is forced out of the socket by bands of cicatricial tissue may be put right by taking a flap from the area just discussed, i.e., the interval between the upper lid and the eyebrow, and passing it through a temporary opening in the upper lid to cover the raw surface left by dividing the bands.

There are several advantages attached to this procedure. In the 
first place, it is far easier to work with a pedicle flap than with an isolated graft. Secondly, the latter very often does not take, whereas it is the exception for the former not to live. And thirdly, the flap itself while retaining these advantages can be kept very thin, so that comparatively little shrinkage will follow.

Healing is allowed to take place for a week before the base is divided and the opening in the lid closed. By this time the flap

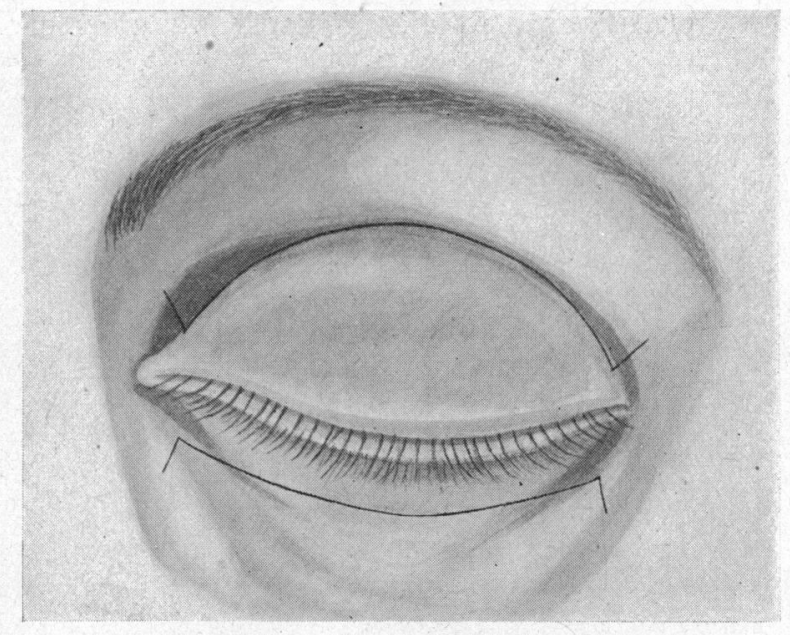

Fig. 5,

has become firmly adherent and is sure to live, and shrinkage has been made good by an additional portion of the pedicle having been drawn into the socket in the process of healing.

\section{(b) Shelving Floor}

An artificial eye sometimes cannot be retained because there is no lower fornix. To remedy the condition a simple operation, such as the "suture" operation of Mr. Thomson Henderson, is in some cases sufficient. But there are others in which something more has to be attempted. For these the following operation is suggested :

A pedicle graft taken from the cheek, slipped into the socket through a temporary opening below the lower lid and anchored to the periosteum (to prevent the new fornix from contracting up) (Fig. 5).

The first skin incision is carried along the infra-orbital margin, At each end of it a vertical cut. is made so as to mark out a skin flap with its base downwards. This flap is carefully undermined and kept as thin as possible without perforating it.

The first incision is now deepened until the floor of the orbit is 
reached, and the wound in the conjunctiva is planned to be so far back as to allow ample conjunctival lining for the lid.

The next step is to suture the skin flap to the posterior lip of the wound in the floor of the orbit. This is done with interrupted catgut sutures and it will be found advantageous to lay all the sutures first and to tie them afterwards.

The first stage of the operation is now complete and the graft is. allowed to heal for a week or ten days.

When this period has elapsed, the vertical cuts at each end of

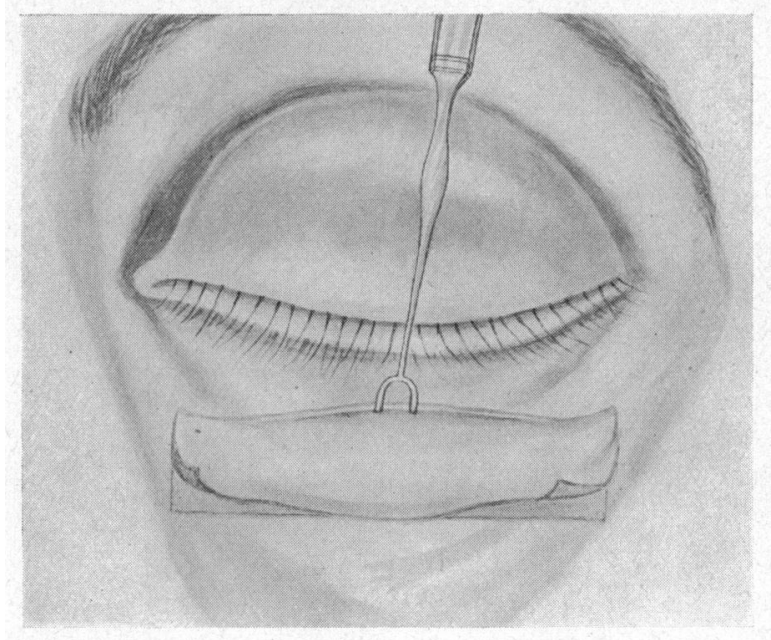

FIG. 6.

the first incision are prolonged a little downwards and their lower ends then joined together by a horizontal incision (Fig. 6). The base of the flap is now partially undermined, turned up and joined to the conjunctiva lining the lid, interrupted sutures being' used as beforc.

It might be thought that all that now remained to be done was to close the wound on the face, and that adhesions of the flap would be sufficient to maintain the depth of the fornix. That is not so. The structures underlying the graft are all too pliable and it is necessary to anchor it to the periosteum. It is this factor in the operation more than any other which, I think, makes for success.

\section{III.-Enlarged Socket}

We are familiar with sockets in which the artificial eye is forced out by the weight of the soft tissues of the roof, and sockets which are so large that the space behind the artificial eye requires filling up with a vulcanite or gutta percha cast of the cavity. Cases of this description may be dealt with by the operation just described 
but applied to the upper lid. The effect of the operation here is to fill up the cavity with a soft pad covered with skin, which forms a support for the artificial eye. The first incision (Fig. 5) is made in the principal fold between the upper lid and the loose tissue above, and the flap is marked out with a short vertical cut upwards at each end of the incision. The flap is then dissected up as before and the original incision deepened until the conjunctiva of the upper fornix is reached. Then the flap is sutured to the posterior lip of the wound in the conjunctiva. A week or ten days afterwards the base of the flap is divided, the deep structures being included in the incision. The margin of the base is then slightly undermined, and sutured to the conjunctiva lining the lid. The flap and the tissues underneath it having lost their support above owing to division of the deeper structures, sink down behind the upper lid in the course of the next week or so.

Patients who require a vulcanite support behind the artificial eye always give it up sooner or later. The reasons are, that the socket alters its shape and the eye does not maintain its original position. In addition to this it is at the best of times a troublesome device. So it is discarded and a shade is worn to hide an empty socket and a sunken lid.

The operation enables the patient to wear an artificial eye in comfort without a special support, and although ptosis results, it is easily remedied as far as the position of the lid is concerned; and the patient can move about freely without drawing particular attention to himself.

\section{ANGIOMA OF THE CHOROID.}

BY

E. ERSKINE HENDERSON, LONDON.

THE interesting case recorded by Paton and Treacher Collins in last year's Transactions of the Ophthalmological Society (Vol. XXXIX, p. 157, 1919) induces me to record another case of what may turn out to be a similar condition, although the eye does not at present show any sign of coming under the pathologist.

The patient, E. H., a girl of eight, was first seen in 1917 on account of convergent strabismus of the left eye. This eye, which was considerably more hypermetropic than its fellow, had no central fixation and was quite amblyopic. The other eye had normal vision. The left side of the forehead, part of the eyelid, and temporal region were covered by a port wine naevus. The fundus at that time appeared normal. I' saw her again at the beginning of 1920 . 